\title{
Hacer el pensamiento visible para el fortalecimiento de las habilidades comunicativas en inglés de niños de transición ${ }^{1}$
}

\section{Making Thinking Visible to Strengthen the Communication Skills in English of Transition Children}

\author{
Sebastián Fernando Marín-Hine ${ }^{2}$ \\ María Nuria Rodríguez de Martínez ${ }^{3}$
}

Citation/ Para citar este Artículo: Marín-Hine, S. y Rodríguez, M. (2021). Hacer el pensamiento visible para el fortalecimiento de las habilidades comunicativas en inglés de niños de transición. Colomb. Appl. Linguistic. J., 23(1), pp. 49-62.

Received: 16-Aug.-2020 / Accepted: 05-May.-2021

DOI: https://doi.org/10.14483/22487085.16818

\section{Resumen}

Esta investigación tuvo el propósito de indagar por el fortalecimiento de las habilidades comunicativas en inglés de niños del nivel de transición en un colegio privado de la ciudad de Bucaramanga (Colombia); nivel que, de acuerdo con el Ministerio de Educación Nacional de Colombia (Decreto 3870 de 2006), es la oferta educativa para los niños de 5 años cumplidos. El planteamiento de este proyecto se abordó desde el diseño de una secuencia didáctica, utilizando como base teórica, por un lado, el enfoque de Pensamiento Visible para el desarrollo y la promoción de disposiciones de mente a través de la implementación de rutinas de pensamiento y, por el otro, el enfoque Natural de la Lengua para el trabajo de la competencia comunicativa. La recolección de datos se hizo a partir de la documentación generada desde las rutinas de pensamiento, los apuntes en el diario de campo y el material fotográfico y audiovisual. Los datos recolectados mostraron el vínculo que existe entre el lenguaje y la producción de pensamiento. Los hallazgos obtenidos fueron: primero, la lengua materna tiene un papel importante en la adquisición de una lengua extranjera. Segundo, el proceso de lectura y escritura en una segunda lengua está estrechamente relacionado con la competencia que tenga el individuo en su lengua nativa. Tercero, las rutinas de pensamiento funcionan como herramientas de andamiaje en la interiorización de vocabulario y en la comprensión de estructuras gramaticales.

Palabras clave: adquisición del lenguaje, lengua extranjera, pensamiento, pensamiento visible, primera infancia

\begin{abstract}
The purpose of this research was to strengthen the communication skills in English of transition children in a private school in Bucaramanga (Colombia). The transition level according to the Ministry of National Education of Colombia (Decreto 3870 de 2006) is the educational offer for children 5 years of age or older. The development of this project was approached from the design of a didactic sequence, using as a theoretical basis the Visible Thinking approach for the development and promotion of mind dispositions through the implementation of Thinking routines, as well as the Natural Language approach for the development of the communicative competence. The data collection was made from the documentation generated from the thinking routines, notes in the field diary, photographic and audiovisual material. The data collected show the link

1 Este artículo es el resultado de una investigación en el aula diseñada como trabajo de grado para la Maestría en Educación de la Universidad Autónoma de Bucaramanga (UNAB), Colombia.

2 Universidad Autónoma de Bucaramanga (UNAB), Colombia. ORCID (D): https://orcid.org/0000-0002-4608-0785. smarin63@unab.edu.co

3 Universidad Autónoma de Bucaramanga (UNAB), Colombia. ORCID (D): https://orcid.org/0000-0001-7050-9484. mrodriguez4@unab.edu.co
\end{abstract}


between language and the production of thought, seeing the acquisition of the English language as a functional tool in the improvement of the mother tongue. The research revealed four findings: First, the mother tongue plays an important role in the acquisition of a foreign language. Second, the process of reading and writing in a second language is closely related to the competence that the individual has in their native language. Third, the thinking routines work as scaffolding tools in the internalization of vocabulary and understanding of grammatical structures.

Keywords: language acquisition, foreign language, thinking, visible thinking, early childhood

\section{Introduction}

Con la apertura de los mercados y la cada vez más difusa línea entre fronteras, la globalización como proceso económico, social, tecnológico, cultural y político integra a los países en una interdependencia de intercambio social basada en la comunicación (Flores, 2016). En este contexto, desde los años 70 el inglés ha ido consolidándose como lengua universal por múltiples razones históricas, políticas y demográficas, convirtiéndose en la lengua oficial de la ciencia e incluso suscitando que grandes compañías multinacionales se radicaran en países angloparlantes, por ejemplo, en los Estados Unidos o en el Reino Unido (BBC News Mundo, 2019). El portal alemán de estadísticas Statista (2020) afirma que en 2019 el inglés fue el idioma más utilizado en el mundo con 1' 268.000 .000 de hablantes nativos, seguido por el mandarín con 1'120.000.000. Esto explica que, con el fin de hacer parte del mercado internacional, los gobiernos de los países no angloparlantes promuevan programas que apunten a desarrollar y fortalecer habilidades comunicativas en inglés.

Colombia, no siendo ajena a esta apertura, adoptó en 2006 el Marco Común Europeo de Referencia para las Lenguas (MCERL) bajo el Decreto 3870 (2006), el cual modificó los artículos 21 y 22 de la Ley General de Educación (Ley 115 de 1994), evidenciando así una preocupación palpable por fomentar el aprendizaje de una nueva lengua (Decreto 3870 de 2006). Los esfuerzos hechos desde el Ministerio de Educación Nacional (MEN) -bajo el mandato de varias administracionesse cristalizaron en la formulación de diferentes programas que buscaron desarrollar habilidades comunicativas en inglés desde el ciclo de educación primaria y siguiendo los lineamientos evaluativos del MCERL, pero sin grandes resultados, tal como lo evidenció el estudio hecho por la compañía sueca EF Education First en 2019. Dentro de la novena edición de la prueba anual EF EPI (English Proficiency Index), que mide el nivel de aptitud en inglés de diferentes países no angloparlantes alrededor del mundo, Colombia ocupó el puesto 68 entre 100 países y el puesto 17 entre los 19 de la región, ubicándose en un nivel de aptitud "bajo" por tercer año consecutivo y ascendiendo lentamente del nivel "muy bajo" en el que se encontraba desde 2011 (EF Education First, 2019). Los resultados de este ranking conllevan a una urgente reflexión alrededor de la eficiencia de las metodologías utilizadas para la enseñanza de este idioma; metodologías que han estado implementándose por más de 14 años y aún distan mucho del nivel de aptitud de países como Argentina, Costa Rica y Uruguay, que ocuparon los tres primeros puestos de la lista a nivel latinoamericano, respectivamente. En el artículo publicado por el periódico El Tiempo (2019), titulado: "Colombia, entre los países que se rajan en el dominio del inglés", haciendo referencia a los resultados revelados por EF, se afirma que "los colombianos [...] no cuentan con las suficientes habilidades comunicativas para, por ejemplo, hacer negocios en ese idioma" y que de acuerdo con Giorgio lemmolo, Director de Gestión Académica de EF Education First, citado en ese mismo texto, parte del problema radica en la "falta de estrategias a largo plazo en torno a la capacitación docente y la implementación de metodologías comunicativas" (El Tiempo, 2019).

Dado lo anterior, el presente artículo hace referencia a los resultados obtenidos tras la implementación de una secuencia didáctica que fue diseñada teniendo en cuenta el enfoque de Pensamiento Visible y que integró rutinas de pensamiento para el fortalecimiento de las habilidades comunicativas en inglés de estudiantes del nivel de transición. La investigación realizada combinó los procesos de adquisición de una segunda lengua con un enfoque metacognitivo, 
siendo la metacognición, de acuerdo con Gonzáles (1996), un conjunto de actividades y funciones cognoscitivas que le permiten a la persona controlar, conocer y autorregular su funcionamiento cognitivo. El enfoque metacognitivo utilizado fue el de Pensamiento Visible que, además de facilitar dicha adquisición lingüística, logró promover el desarrollo de habilidades de pensamiento en los estudiantes. A continuación, se da cuenta de la importancia del inglés como lengua extranjera en el país, la necesidad de promover la enseñanza de una segunda lengua desde la primera infancia y la efectividad de las actividades diseñadas bajo el enfoque de Pensamiento Visible para la comprensión del lenguaje.

La búsqueda de metodologías que pongan a la comunicación como fin último de la enseñanza del inglés se hace urgente al examinar los resultados presentados por la EF, pues se requiere que contribuyan al fortalecimiento de habilidades comunicativas en ese idioma, pero que también tengan en cuenta el desarrollo cognitivo del individuo tal como lo confirman los numerosos estudios que vinculan la génesis del pensamiento con la aparición del lenguaje (Castaño, 2003; Damasio y Damasio, 1992; Verdejo-García y Bechara, 2010). Visto así, la enseñanza de una lengua extranjera debería constituirse en una herramienta para el desarrollo del pensamiento.

Hablar de desarrollo, a su vez, implica necesariamente una revisión a la primera infancia, etapa crucial en la vida de todo ser humano para el aprendizaje y la adquisición del lenguaje (Kuhl, 1998; Mayoral, 2016; Piaget, 2016; Vygotsky, 2015). Durante los primeros meses de vida el individuo utiliza el lenguaje corporal y gestual como herramienta para la comunicación con su entorno y, posteriormente, alrededor de los 12 meses, de acuerdo con Gopnik et al. (1999), incursiona en el lenguaje hablado, que se convertirá en el soporte psicológico intermental e intramental con el que desarrollará procesos mentales superiores. Vygotsky (1978) reconoce dentro de los procesos mentales superiores capacidades como las gnosias, praxias, atención, lenguaje, toma de decisiones, razonamiento, planificación e inhibición; procesos que más adelante Azcoaga (1974) identifica como necesarios para el aprendizaje mismo, denominándolos "Los Dispositivos Básicos de Aprendizaje".

Es así como este artículo se propone mostrar los resultados obtenidos del proyecto de investigación de corte cualitativo, que buscó fortalecer las habilidades comunicativas en inglés de niños del nivel de transición en un colegio privado de la ciudad de Bucaramanga (Colombia), utilizando el enfoque de Pensamiento Visible para desarrollar habilidades de pensamiento a medida que se promueve la adquisición de la nueva lengua.

\section{Marco teórico}

\section{El niño de preescolar: una visión constructivista}

Dentro de las múltiples teorías del aprendizaje y corrientes del pensamiento que se han desarrollado a lo largo de la historia, tal vez uno de los enfoques que cuenta con mayor aceptación y popularidad en las últimas décadas es el constructivismo. Para autores como Abbotty Ryan (1999), y Méndez (2002), la construcción del conocimiento en el ser humano ocurre por la constante interacción del sujeto con su entorno, en donde cada persona organiza, percibe y le da sentido a la realidad en forma de constructos que incorpora a sus conocimientos previos gracias a la actividad de su sistema nervioso, y que genera, en consecuencia, nuevos conocimientos; a este proceso se le denomina "constructivismo". Son representantes de este enfoque teóricos tan influyentes como el epistemólogo suizo Jean Piaget, el psicólogo ruso Lev Vygotsky y los psicólogos estadounidenses Jerome Bruner y David Ausubel, entre otros.

El conocimiento, al ser una construcción genuina del sujeto y que no se da únicamente a través de la experiencia, tal como lo proponían los empiristas, y tampoco al ser una copia de ideas del mundo externo reflejadas a modo de espejo, como lo concibió Hume (2005), ni mucho menos un despliegue de conocimientos innatos inamovibles, es, entonces, una fabricación realizada por el individuo, quien modifica sus conocimientos 
gracias a la interacción con el entorno, no estando completamente constreñido por las características del medio ni por sus determinantes biológicos (Serrano y Pons, 2011).

Uno de los grandes estudiosos en el campo del desarrollo infantil fue sin duda Piaget (2017), que con su teoría epistemológica logró vislumbrar una serie de etapas en las que el niño, desde su nacimiento, avanza a medida que su sistema nervioso y los órganos de sus sentidos van madurando (Piaget, 2016). La concepción de dichas etapas o periodos del desarrollo han estado bajo constante revisión y discusión, como da cuenta Gardner (2011), quien afirma que el esquema Piagetano, a pesar de ser el mejor con el que contamos actualmente, muestra unas deficiencias específicas y evidentes a la luz de nuevos estudios. Dicho lo anterior, Gardner (2011) también resalta la relevancia que tiene el trabajo de Piaget en torno al desarrollo de ciertas habilidades logradas por los niños, como lo son la adquisición del lenguaje dentro de la etapa pre operacional y el estudio del desarrollo de la inteligencia lógicomatemática. De ahí que el trabajo de Piaget (2018) resulte de gran importancia al momento de generar un mapa de comprensión en torno al desarrollo del niño perteneciente a la etapa pre operacional, con edades entre los 5 y 6 años.

\section{El desarrollo del pensamiento y el lenguaje}

El rol que tiene la cultura para el desarrollo del pensamiento, así como la colaboración entre el individuo y la sociedad, es una de las características principales dentro de lo que Vygotsky (1978) denomina "la teoría sociocultural", en la que la interacción social transforma la actividad práctica considerando los instrumentos y símbolos que median en este proceso desde una perspectiva evolutiva, siendo el conocimiento una construcción social (Suárez, 2014). A diferencia de Piaget, quien veía en el nacimiento de la inteligencia un proceso individual y dependiente de lo biológico, Vygotsky propone una nueva perspectiva en la cual el factor social es la clave para el aprendizaje del sujeto, prestando particular atención al desarrollo del lenguaje, visto como una herramienta psicológica que posteriormente adquiere el status de función mental superior, permitiendole al individuo interactuar con el medio y apropiarse de la riqueza cultural del conocimiento (Vygotsky, 2015). Estos instrumentos psicológicos, tales como los sistemas de lenguaje, signos y gestos, las capacidades mnemotécnicas y los sistemas de toma de decisiones, son construcciones artificiales que poseen una orientación interna y buscan "transformar las aptitudes y destrezas de la naturaleza humana en funciones mentales superiores" (Vygotsky, 2015, p. 23).

La teoría sociocultural, por lo tanto, nos permite una mejor comprensión del rol social como epicentro del aprendizaje; en ella, la mediación entre individuos es de vital importancia para la construcción del pensamiento, siendo el lenguaje la herramienta que permite el proceso de internalización de la actividad social (interpersonal) a la actividad interna (intrapersonal), alcanzando así el desarrollo de las funciones superiores.

Ahora bien, en el caso de la enseñanza de una lengua extranjera bajo el enfoque Natural de la Lengua es el profesor quien asume el rol de guía y mediador a manera de modelo de la lengua nueva, así como el de creador de una atmósfera propicia para la adquisición de esta lengua y, finalmente, es el principal encargado de presentar y organizar los contenidos en una serie de actividades dentro del salón de clases (Krashen y Terrell, 1998; Richards y Rodgers, 2014). Este proceso de mediación entre profesor-estudiante descrito por Krashen y Terrel (1998) utiliza la propuesta de Vygostky (2018) denominada la "Zona de Desarrollo Próximo" (ZDP), definida como

la distancia entre el nivel real de desarrollo, determinado por la capacidad de resolver independientemente un problema, y el nivel de desarrollo potencial, determinado a través de la resolución de un problema bajo la guía de un adulto o en colaboración con otro compañero más capaz. (Vygotsky, 2018, p. 133)

Las interacciones juegan entonces un papel importante en el desarrollo cognitivo del niño, tal como lo indica Dehaene (2019) en su estudio acerca de cómo aprende el ser humano, pues visto desde la neurociencia se ha comprobado que durante los 
primeros años de vida el cerebro del niño presenta una gran capacidad para aprender a través de la neuroplasticidad, que le permite al sistema nervioso generar conexiones y cambiar su estructura como respuesta a los estímulos del entorno. Y es que el entorno no solo influye en la génesis del pensamiento sino también en la adquisición del lenguaje, tal como proponen Hart y Risley (1995), quienes demostraron cómo el entorno sociocultural afecta el desarrollo del lenguaje oral. Dicho estudio demostró que las familias con menos recursos económicos producen 616 palabras por hora, mientras que las familias de clase trabajadora producen 1251 y en las familias de padres con formación universitaria la cifra asciende a 2153.

\section{El niño de transición y la adquisición de una lengua extranjera}

A pesar de los múltiples estudios llevados a cabo en torno a la importancia que tiene la primera infancia para el aprendizaje y el desarrollo del lenguaje, no es sino hasta el 2016 cuando el Ministerio de Educación Nacional (MEN) incluye dentro de los lineamientos para la enseñanza del inglés como lengua extranjera -hasta el momento regulados únicamente a partir de la primaria- los DBA (Derechos Básicos de Aprendizaje) y el currículo sugerido de inglés para el nivel de transición, dejando por fuera al resto del preescolar, como lo apuntan Pérez y Villamizar (2018). Sin embargo, con el fin de aprovechar al máximo la capacidad de aprendizaje que poseen los niños en los primeros años, se hace necesario plantear la enseñanza de una lengua extranjera desde la edad más pronta posible teniendo en cuenta los procesos naturales de adquisición (Fleta, 2006; Kuhl, 1998, 2010; López y Muñoz, 2003; Mayoral, 2016; Navarro, 2010; Reyes, 2005).

En este contexto, la presente investigación tuvo como objetivo general indagar por el fortalecimiento de las habilidades comunicativas en inglés, motivo por el cual el constructo teórico y metodológico se basó en el enfoque comunicativo para la enseñanza de la lengua (CLT: Communicative Language Teaching). Este enfoque busca hacer de la competencia comunicativa el objetivo principal de la enseñanza de idiomas y, con ello, desarrollar procedimientos para la adquisición de las cuatro habilidades lingüísticas (escuchar, hablar, escribir y leer) que den cuenta de la interdependencia del lenguaje y la comunicación (Richards y Rodgers, 2014).

Como parte del CLT aparece el enfoque Natural de la Lengua propuesto por Krashen y Terrell (1998). En este enfoque el aprendizaje está centrado en la comprensión de estructuras (mensajes) llamados "input", presentados bajo la fórmula $i+1$, en donde $i$ (input) es el nivel de competencia actual del estudiante $y+1$ la etapa siguiente dentro del proceso natural de la adquisición de la lengua. Krashen y Terrell (1998), al igual que Vygotsky (2015), ven en la adquisición de una nueva lengua procesos similares a los observados en la adquisición de la lengua materna. En ambos casos el estudiante avanza por una serie de etapas en las que intervienen factores emocionales y actitudinales, cierta cantidad de input comprensible en mayor o menor grado, la diferencia de edad y la aptitud hacia una nueva lengua.

Respecto a la enseñanza de una lengua extranjera en la escuela, Vygotsky (2015) afirma que el conocimiento avanzado de la propia lengua juega un papel importante en el estudio del idioma extranjero, dado que al aprenderlo utilizamos significados de palabras que ya fueron desarrolladas en la lengua nativa: "la adquisición de lenguas extranjeras y nativas pertenece a la misma clase de procesos del desarrollo del habla" (Vygotsky, 2015, p. 226).

Esta perspectiva de adquisición natural del lenguaje que vincula a la lengua materna con la lengua extranjera permite asignarle al lenguaje nativo del niño un valor importante dentro de su aprendizaje. El trabajo llevado a cabo por Salmon (2008) en Miami (Estados Unidos), y que sirve de antecedente para este proyecto, demostró que los estudiantes de preescolar provenientes de familias no angloparlantes mejoraron su preparación lingüística al adquirir un segundo idioma con mayor facilidad sin sacrificar su lengua materna; esto también tuvo un impacto positivo en los niveles de participación de la clase durante los procesos de interacción entre lenguaje y cognición. Para ello Salmon (2008) utilizó rutinas de pensamiento como herramienta pedagógica. En 2016 Dajani logró resultados similares en Palestina al comprobar una 
mejoría de las habilidades de comunicación en inglés si, además, se les permitía a los estudiantes (de cuarto y quinto de primaria) expresarse en su lengua materna durante las rutinas de pensamiento, argumentando que esto les otorgaba una mayor confianza en sus habilidades, abría sus mentes y los enriquecía intelectualmente (Dajani, 2016).

De esta manera, la adquisición de una lengua extranjera es vista como una herramienta social que se logra de forma natural a través de la interacción del individuo con otros sujetos y que a su vez puede contribuir al desarrollo del pensamiento del estudiante.

\section{Pensamiento visible}

El enfoque de Pensamiento Visible (Visible Thinking), que apareció en 2016, hace parte del proyecto educativo Proyecto Zero (PZ: Proyect Zero $)^{4}$ fundado en 1967 por el filósofo Nelson Goodman en la Escuela de Educación de Graduados de la Universidad de Harvard. Como proyecto investigativo, Pensamiento Visible es el resultado de cinco años de exploración cuyo objetivo ha sido encontrar formas de cultivar lo que Ritchhart (2002) denominó "disposiciones de pensamiento" dentro del ámbito escolar (Ritchhart et al., 2011).

Para Ritchhart (2002), investigador senior de este proyecto, el pensamiento es un proceso desordenado, complejo, dinámico e interconectado, y no un desarrollo secuencial ni sistemático; va mucho más allá de lo propuesto dentro de la taxonomía de Bloom y Krathwohl (1956) y su desarrollo debe ser prioridad dentro de los procesos de enseñanza y aprendizaje en todas las escuelas (Perkins, 1992).

Con el fin de desarrollar habilidades de pensamiento, Pensamiento Visible propone una serie de rutinas llamadas "rutinas de pensamiento". Estas son herramientas diseñadas para promover el pensamiento y, de acuerdo con Ritchhart et al. (2011), a través de unos pocos pasos ayudan a centrar la atención en los diferentes procesos del pensamiento, siendo de gran ayuda en la

4 Para consultar el proyecto, dirigirse a: http://www.pz.harvard. edu/50th construcción del entendimiento. Las rutinas de pensamiento se pueden ver desde tres perspectivas diferentes: a) como herramientas, b) como estructuras y c) como patrones de comportamiento (Ritchhart et al., 2011).

Esta última perspectiva tiene gran importancia dentro de un salón de clases, donde las rutinas de pensamiento son las que dominan las actividades de un grupo. Para Ritchhart, una rutina puede ser pensada como un patrón de comportamiento que muestra la forma en la que un grupo trabaja:

\section{Lo que parece suceder sin esfuerzo en aulas bien gestionadas es realmente el resultado del establecimiento de rutinas [...]. Debemos también establecer rutinas de aprendizaje y de pensamiento en nuestros salones que ofrezcan a los estudiantes estructuras que les permitan proceder y herramientas que puedan controlar y usar para su propio aprendizaje. (2015, p. 9)}

Dentro del proyecto de Pensamiento Visible, Ritchhart et al. (2011) presentan un compilado de 21 rutinas de pensamiento organizadas dentro de tres grandes categorías: presentar y explorar, sintetizar y organizar, y explorar ideas a profundidad.

Para el caso de la presente investigación se utilizaron las rutinas pertenecientes a la primera categoría: presentar y explorar ideas, sin que esto haya sido una decisión restrictiva que dejara por fuera al resto de las rutinas, pues, aunque se realizaron con menor frecuencia y nivel de complejidad, también formaron parte importante de la dinámica de la clase.

Adicional a lo anterior, Ritchhart (2015) también propone ocho fuerzas culturales presentes dentro de las "culturas de pensamiento" (CoT: Cultures of Thinking), definidas por el autor como lugares en donde el pensamiento individual y colectivo es valorado, visible y promovido a diario a través de la interacción de todos los miembros del grupo. Para Ritchhart la cultura es la llave de la transformación y es por eso que, como promotores del pensamiento, es indispensable comprender y trazar un marco con el que podamos entender y mejorar la forma en que aprendemos: 
La cultura de pensamiento no hace referencia a un kit de prácticas o a una expectativa general de que las personas deberían estar involucradas en el pensamiento. Una cultura de pensamiento produce los sentimientos, la energía e incluso la dicha que puede impulsar el aprendizaje y nos motiva a realizar ese trabajo mental que algunas veces puede parecernos difícil y desafiante. (2015, p. 5)

Como resultado de su investigación, Ritchhart (2015) identifica las siguientes fuerzas culturales que todo maestro debe promover en el aula, buscando el desarrollo de estudiantes con gran capacidad de pensamiento y aprendizaje: expectativas, lenguaje, tiempo, modelaje, oportunidades, rutinas, interacciones y ambiente.

Por eso al hablar de Pensamiento Visible no se hace referencia solamente al uso de las rutinas de pensamiento como herramientas descontextualizadas. De ahí que el estudio presentado aquí, y que tuvo como objetivo principal desarrollar habilidades comunicativas en inglés, tomara este enfoque como eje principal dentro de la construcción de la estrategia pedagógica en la que el entorno, el contexto, la metacognición y la comprensión fueron elementos claves durante el proceso.

Desde esta investigación se tuvo la convicción de que la enseñanza se debe dar de tal forma que el estudiante sea, además del centro del proceso, un individuo autónomo, consciente de su propio desarrollo cognitivo y que, más allá de la memorización de contenidos, pueda desarrollar herramientas que le permitan construir, tal como expone Bruner et al. (1987) con su teoría del andamiaje, las estructuras de su propio pensamiento.

Al respecto, el neurocientífico cognitivo Dehaene (2019) afirma que desde la neurociencia se ha podido evidenciar la eficacia de intervenciones pedagógicas que apuntan a la metacognición, ya que saber aprender es uno de los factores más importantes del éxito escolar. ${ }^{5}$

5 Esto puede corroborarse en la página oficial de Education Endowment Foundation (EEF), en la que se relacionan listas de las más exitosas intervenciones pedagógicas.

\section{Metodología}

Este proyecto de investigación se ubica dentro de la metodología investigación-acción. Elliot (1993) define la investigación-acción como el estudio de una situación social para tratar de mejorar la calidad de la acción en la misma. En cuanto al planteamiento de la investigación, se buscó fortalecer las habilidades comunicativas en inglés de niños del nivel de transición a través de la implementación de una secuencia didáctica basada en el enfoque de Pensamiento Visible, con el fin de vincular la adquisición del lenguaje con el desarrollo del pensamiento.

La intervención de corte cualitativo tuvo lugar en un colegio privado para la primera infancia ubicado en la ciudad de Bucaramanga (Colombia), en la que participaron 15 estudiantes del nivel de transición con edades entre los 5 y 6 años. Cabe resaltar que en la institución se implementa el currículo internacional propuesto desde el modelo VESS en el que, además del enfoque de Pensamiento Visible, confluyen diferentes teorías en torno al desarrollo y la utilización del pensamiento como herramienta pedagógica, que son materializadas en diferentes módulos a modo de proyectos.

\section{Recolección de la información}

Para la recolección de la información se utilizó la técnica de observación participante definida por Taylor y Bogdan (1984) como el ir y venir de los observadores entre los datos recogidos y el campo. Esta técnica de recolección fue elegida por la misma naturaleza de la intervención y la población intervenida. También se utilizó el registro de notas en el diario de campo, dado que le permite al investigador un monitoreo permanente del proceso de observación (Bonilla y Rodríguez, 1997).

Fueron importantes, también, las producciones escritas de los estudiantes -resultantes de la documentación dentro de las rutinas de pensamiento-, las fotografías de las rutinas de pensamiento y las transcripciones de diferentes interacciones entre profesor-estudiante y estudianteestudiante. Es necesario mencionar en este apartado 
la importancia de la documentación dentro de las rutinas de pensamiento como una herramienta adicional para la recolección de datos, ya que el objetivo de esta fue registrar las diferentes ideas de los estudiantes a lo largo del tiempo para poder hacer un análisis tanto del proceso de enseñanzaaprendizaje como del avance de cada uno de los participantes.

\section{Problema de investigación}

La enseñanza del inglés dentro de las instituciones promueve el desarrollo de habilidades comunicativas a través de procesos tradicionales de repetición y memoria, en los que se privilegia la escritura y la lectura con el objetivo de que, al finalizar el nivel de transición, los estudiantes puedan responder adecuadamente las pruebas diagnósticas de acceso a la educación primaria en colegios bilingües o que tienen un mayor énfasis en la lengua inglesa. Este tipo de pruebas generalmente son escritas y únicamente requieren que el estudiante pueda relacionar palabras con dibujos, sujetos con verbos y, en mayor o menor medida, un amplio banco de vocabulario referente a: partes de plantas, figuras geométricas, números, colores y animales. La parte oral de las pruebas (si aplica) consiste en una serie de preguntas estandarizadas que son respondidas por el estudiante utilizando estructuras fijas mecánicas y que poco o nada requieren de un proceso de comprensión por parte del niño; motivo por el cual, a pesar de reconocer las palabras y desempeñarse bien en este tipo de pruebas de admisión, el estudiante egresado de dicha institución no puede hacer uso de la lengua extranjera como herramienta de comunicación.

Sumado a este problema, está la falta de alineación del curso de inglés con la cultura de pensamiento en la que se encuentran inmersas las instituciones. Lo anterior puede tener diferentes razones, como la falta de un currículo diseñado para el área, la rotación de profesores de inglés y, en mayor medida, la concepción de que el inglés es una asignatura más, necesaria para cumplir un requisito, que no forma parte integral de la formación del niño.
Con base en lo anterior, este proyecto de investigación se planteó dos preguntas:

- ¿Cómo fortalecer las habilidades comunicativas en inglés de los niños de transición de un colegio privado en la ciudad de Bucaramanga (Colombia), con actividades que a su vez ayuden a desarrollar habilidades de pensamiento?

- ¿Cómo diseñar actividades que promuevan el fortalecimiento de las habilidades comunicativas en inglés, pero que no dependan de un contenido específico?

\section{Diseño de la secuencia didáctica y las categorías de análisis}

Para esta intervención se diseñó una secuencia didáctica teniendo en cuenta los planteamientos vistos a la luz de dos enfoques: por un lado, el uso de rutinas de pensamiento para la activación de habilidades de mente y, por el otro, el enfoque Natural de la lengua propuesto por Krashen y Terrell (1998) para la enseñanza del inglés como lengua extranjera. Como lo plantea Díaz-Barriga (2013), una secuencia didáctica es entendida como "la organización de las actividades de aprendizaje que se realizarán con los estudiantes y para los estudiantes con la finalidad de crear situaciones que les permitan desarrollar un aprendizaje significativo" (p. 1).

La secuencia didáctica diseñada contó con 15 actividades que giraron en torno a la comprensión del cuerpo, donde el eje temático "My body" (Mi cuerpo) fue enteramente circunstancial pues hizo parte de los contenidos propuestos por la institución para el periodo en el que se realizó la intervención.

De forma semanal se desarrolló una actividad de la secuencia didáctica a la par que se realizaban otras actividades que ya estaban establecidas desde el plan de área, como por ejemplo el trabajo en el libro y el desarrollo de guías que, a pesar de no hacer parte de la intervención, sirvieron de ejercicios complementarios.

En la Tabla 1 se muestran las diferentes categorías y subcategorías tenidas en cuenta para 
el análisis del fortalecimiento de las habilidades comunicativas en inglés. Estas surgieron teniendo como base el nivel de referencia A1 (acceso) propuesto desde el MCERL.

\section{Análisis: razonando con evidencia}

Para el análisis de esta intervención pedagógica se tuvieron en cuenta los diferentes desempeños mostrados por los estudiantes durante cada una de las actividades dentro de la secuencia didáctica. Los datos recogidos sistematizados dentro de rúbricas, producciones escritas y transcripciones de conversaciones se compararon con los obtenidos durante el diagnóstico, en el que se realizaron tres sesiones con dos rutinas de pensamiento: "Zoom In" y "I see, I think".

En el diagnóstico se evidenció que la producción oral estaba limitada a respuestas no verbales de preguntas simples, ya sea por la no comprensión de la indicación dada por el profesor, por la falta de herramientas lingüísticas en inglés para hacer declaraciones o por la limitante de no poder usar el español como lengua de apoyo. A su vez, la suma de las diferentes situaciones mencionadas anteriormente condujo a una baja participación durante la primera rutina, hecho que adjudicamos a lo que Krashen y Terrell (1998) denominaron "la teoría del filtro afectivo", es decir, la baja motivación para ser parte de la experiencia de aprendizaje debido a factores emocionales y actitudinales. Durante la segunda rutina del diagnóstico ("I see, I think") se pudo observar que, al aceptar que los estudiantes utilizaran conceptos del español para explicar lo que estaban viendo y lo que pensaban, hubo una mejoría en la participación, logrando que dentro de sus respuestas al menos 3 estudiantes incluyeran el yes (si) y no (no) a preguntas cortas de manera espontánea, así como en la incorporación de nuevas palabras transferidas del español al inglés.

\section{Sobre la comprensión y la producción oral}

Cuando se les permitió a los estudiantes -de forma implícita- utilizar el español, las interacciones comenzaron a cambiar de acuerdo con la direccionalidad del discurso. Así, identificamos 5 momentos diferentes dentro de las sesiones.

El primer momento, que ocurre justo después de la instrucción inicial, es aquel en el cual el estudiante dialoga consigo mismo y trata de darle sentido a lo que el profesor acaba de decir. Este diálogo sucede a veces en voz alta y otras veces en silencio, pero siempre en español, lo que lleva a pensar que la interiorización de los estímulos visuales y auditivos ocurre primero en la lengua materna, sirviendo de base para la construcción de un nuevo concepto en la lengua nueva.

El segundo momento es la interacción entre estudiantes en el instante en que hay una pregunta específica. La conversación ocurre totalmente en español, el individuo contrasta su idea con el grupo confirmándola, complementándola o eliminándolapara ser posteriormente lanzada en forma de respuesta utilizando los recursos lingüísticos que posee. En este punto la comprensión del estudiante aumenta al confrontar su idea con el grupo, por lo que su participación incrementa al haber una motivación por saber y conocer, y esto hace que el filtro afectivo baje y permita la adquisición. Es importante mencionar que el profesor nunca habla en español. Enseguida, un tercer momento se presenta y es la intervención nuevamente del profesor ante la respuesta del estudiante; intervención en la que aporta nuevas estructuras y vocabulario, confirma

Tabla 1. Categorías y subcategorías para el análisis del fortalecimiento de las habilidades comunicativas en inglés.

\begin{tabular}{cc|cc|c}
\hline \multicolumn{5}{c}{ Categorías de análisis } \\
\hline \multicolumn{4}{c}{ Hablar $(\mathrm{H})$} & Escribir (E) \\
\hline Escucha $(\mathrm{CE})$ & Lectura $(\mathrm{CL})$ & Monólogos $(\mathrm{HM})$ & Conversación $(\mathrm{HC})$ & Escritura (EE) \\
\hline
\end{tabular}

Fuente: elaboración propia. 
conceptos u ordena la estructura dada mediante la repetición, sin puntualizar en el error.

Los dos últimos momentos observados (cuarto y quinto) es lo que denominamos "etapas post feedback" y son las nuevas ideas en forma de pensamiento individual que se añaden a la nueva estructura pero que esta vez no pasan por la socialización grupal entre pares, sino que se presentan a modo de respuesta inmediata, ahora en inglés y con tendencia a reducir el error. Posteriormente, la validación de estructuras y la constante exposición al vocabulario objetivo reemplazan y acomodan la producción oral del individuo siempre y cuando le sea necesario hacer uso de la nueva lengua. Sin embargo, también se observó que el estudiante únicamente cambia su discurso cuando tiene que hacerlo, ya que, a pesar de interiorizar nuevas palabras y estructuras, solo hace uso de ellas cuando el entorno le indica que así debe hacerlo, de lo contrario la lengua materna siempre va a ser su opción segura; lo anterior se demuestra con las interacciones de clase: mientras el estudiante trabaja con otros compañeros usa el español, pero ante la intervención del profesor u otro agente acomoda su discurso con el fin de poder comunicarse siempre y cuando tenga los recursos lingüísticos para hacerlo.

\section{Acerca del proceso de lectura y escritura}

La documentación hace parte de las rutinas de pensamiento, ya que es allí donde quedan plasmadas las diferentes ideas producidas por los estudiantes y que con el paso del tiempo permiten generar un panorama general sobre el recorrido del pensamiento alcanzado en una historia de aprendizaje. La documentación también juega un papel importante a la hora de dinamizar los procesos de lectura y escritura de los niños en los que, dicho sea de paso, influyen enormemente los desempeños del estudiante en su lengua materna.

Tras la intervención se observó que el uso de rutinas de pensamiento, al menos las utilizadas para esta investigación, no fueron un recurso que promovieran activamente habilidades de escritura por sí solas. A la hora de documentar, los niños se sirvieron de la transcripción de palabras fijas dadas por el profesor que respondían a las estructuras clave a utilizar dentro de la rutina. Por lo demás, la escritura libre se denominó "escritura creativa".

Este proceso de escritura permite que el estudiante utilice grafemas individuales asociados a un sonido con el que posteriormente formará una palabra. Durante la intervención, este fenómeno se hizo evidente principalmente en niños que ya tenían una mayor claridad de su proceso en la lengua materna, pudiendo distinguir entre las propiedades del español y el inglés al momento de escribir en la lengua extranjera. Algunos ejemplos se presentan en la Tabla 2.

En cuanto a la Tabla 3, se puede observar otro tipo de representaciones escritas en donde la fonética del español permea la forma de escribir palabras en inglés sin que haya realmente una conciencia a nivel fonológico de la lengua extranjera.

Además de los acercamientos hechos por los estudiantes en la escritura creativa, también es importante mencionar que con la repetición del vocabulario y la presentación de nuevas estructuras en las que podían reciclar las palabras aprendidas, al final del proceso todos los estudiantes, tanto los del grupo de la Tabla 2 como los de la Tabla 3, lograron una mejoría en la forma de escribir correctamente las palabras de uso común.

Tabla 2. Producciones escritura creativa.

\begin{tabular}{cc}
\hline Escritura creativa & Palabra correcta \\
\hline iex & Eyes \\
\hline teef & Teeth \\
\hline hort & Heart \\
\hline jed & Head \\
\hline
\end{tabular}

Fuente: elaboración propia. 
Hacer el pensamiento visible para el fortalecimiento de las habilidades comunicativas en inglés de niños de transición

Tabla 3. Producciones escritura literal.

\begin{tabular}{cc}
\hline Producción estudiante & Palabra correcta \\
\hline elbou & elbow \\
\hline touz & Toes \\
\hline jan & Hand \\
\hline Pinas & Penis \\
\hline
\end{tabular}

Fuente: elaboración propia.

Así mismo, la documentación como herramienta de registro dentro de las rutinas de pensamiento, permitió un proceso de monitoreo, ya que en ella los estudiantes pudieron visitar trabajos previos, comparando procesos y haciendo correcciones gramaticales a medida que las actividades se iban conectando. Incluso aquellos estudiantes a los que aún se les dificultaba escribir en su lengua materna pudieron documentar haciendo uso de dibujos, a los que posteriormente -en la socialización-se les asignó una palabra para que de esta forma se lograra la relación imagen-palabra. A medida que se avanzó en las actividades, los estudiantes que documentaron con dibujos lograron una interiorización consciente del vocabulario mediante el proceso de transcripción.

Sin duda, el fortalecimiento de la habilidad de lectura dentro de las rutinas de pensamiento, al igual que la escritura, es un proceso que está relacionado con los avances del niño en la lengua materna. Aun así, al poder visitar sus producciones en documentaciones previas, la lectura aparece como una herramienta de consulta espontánea que le permite a los estudiantes crear espacios sociales autónomos en torno a cada actividad, pudiendo corregir el trabajo de otros e incluso sus propias ideas, discutir acerca de la estética utilizada y sentirse un miembro activo del proceso de aprendizaje al ver que su trabajo es tenido en cuenta para la construcción de nuevas ideas.

\section{Conclusiones}

El desarrollo de las actividades con el enfoque de Pensamiento Visible permitió el aumento de la comprensión auditiva de instrucciones sencillas y palabras de uso común de los estudiantes, así como la producción oral de frases cortas que dan respuesta a preguntas simples y buscan proporcionar información de otras personas y objetos.

Los resultados obtenidos tras el análisis de la intervención sugieren que el uso de herramientas que permiten la documentación del pensamiento y el progreso del niño, tales como las rutinas de pensamiento y los organizadores gráficos, aportan positivamente al desarrollo de las habilidades comunicativas, ya que dejan ver que el estudiante no solo se conecta mejor dentro de la actividad, aumentando los niveles de participación, sino que también permiten el andamiaje necesario a nivel de contenido y lenguaje gracias a la mediación del docente que actúa como fuente primaria de input comprensible de la lengua extranjera.

Como conclusiones emergentes de este proyecto de investigación se propone:

1. Hacer el pensamiento visible a través de organizadores gráficos y rutinas de pensamiento favorece el desarrollo de las habilidades comunicativas en inglés de los niños de transición al ofrecerles un punto de partida físico (la documentación de las rutinas) que soporte la adquisición de nuevos conceptos, estructuras y vocabulario, restándole prioridad a la memoria como único recurso de almacenamiento de información.

2. Hacer el pensamiento visible a través de la documentación escrita le permite al niño apropiarse de estructuras nuevas mediante la lectura y posterior transcripción, y posibilita la ejecución de estructuras cortas que ya conoce dentro de un patrón específico que ha adquirido 
de forma natural con la ayuda de la repetición. También, gracias al tipo de rutina seleccionada para cada actividad, quedan al descubierto los movimientos de mente utilizados para el desarrollo de las actividades.

3. Mediante el diseño de la secuencia didáctica se evidenció que, al desarrollar actividades que le permiten al estudiante conectarse de múltiples formas con el mensaje (contenido) dado por el profesor (input), además de poder hacer uso de su lengua materna para expresar sus ideas, no solo aumenta su nivel de participación dentro de las actividades, sino que además se enriquece su adquisición del conocimiento, la comprensión del mismo y finalmente la exteriorización de ese pensamiento, utilizando diferentes maneras para documentarlo.

4. Las rutinas de pensamiento y los organizadores gráficos favorecen los procesos de interacción profesor-estudiante y estudiante-estudiante, logrando un desarrollo significativo en la habilidad del habla (speaking), comenzando por el monólogo hasta alcanzar el nivel de conversación sencilla. Dentro de las fases propuestas por Krashen y Terrell (1998) en el Enfoque Natural para la adquisición del lenguaje, se evidenció que las interacciones influyen en el paso de la etapa de pre-producción a la de producción temprana, en la que los estudiantes responden algunas preguntas utilizando palabras sueltas y frases cortas, y también son capaces de rellenar formularios y utilizar patrones fijos de conversación.

5. La propuesta pedagógica basada en hacer visible el pensamiento promovió el fortalecimiento de todas las habilidades comunicativas en inglés, pues a medida que el estudiante interactuaba con el profesor y sus compañeros trabajaba la habilidad de escucha (listening) y habla (speaking), y cuando tuvo que documentar su pensamiento (en el caso de ser escrito) fortalecía la escritura (writing), ya sea transcripción y/o escritura creativa. Finalmente, al tener que visitar la documentación para retomar ideas previas y plantear nuevas, el estudiante hizo uso de la habilidad de lectura (reading).
Con respecto a la lectura y escritura en inglés, se evidenció que el avance de estas dos habilidades está directamente relacionado con los mismos procesos llevados por el niño en su lengua materna, sin que esta afirmación sugiera que, porque un estudiante sea capaz de leer en español, automáticamente va a leer en inglés. Lo que este proyecto de investigación permitió observar es que cuando un estudiante lleva un buen proceso de lectura y escritura en español se le facilitará el acceso al material escrito en inglés, dependiendo del nivel de reconocimiento fonético de la lengua extranjera que posea.

La implementación de esta estrategia pedagógica dio cuenta de la importancia de privilegiar el desarrollo de las habilidades comunicativas en inglés a través de la promoción de procesos mentales y de pensamiento dentro de las clases, logrando ir más allá del aprendizaje memorístico, generalmente utilizado para la enseñanza del inglés y que no garantiza realmente la adquisición del lenguaje. Teniendo los resultados como evidencia, ahora se nos plantea un nuevo reto: la búsqueda de nuevas formas para hacer del aprendizaje del inglés en el nivel de transición un proceso más significativo, con sentido y contextualizado, que reemplace o complemente al modelo tradicional basado en la memoria y la gramática como eje fundamental.

Alineados con la idea de Perkins (1992) sobre la búsqueda de nuevos métodos de enseñanza, pensamos que una experiencia de aprendizaje del inglés que busque fortalecer las habilidades comunicativas a través del pensamiento no necesita plantear una nueva metodología de instrucción sino enriquecer aquellos enfoques que ya tenemos, como el enfoque Natural de la Lengua, pensando en llevarlos más allá del contenido.

\section{Aportes a la enseñanza del inglés como lengua extranjera}

Quizá uno de los aportes a la metodología de la enseñanza del inglés como lengua extranjera es la posibilidad de vincular las herramientas propuestas desde el enfoque de Pensamiento Visible para el desarrollo de las cuatro habilidades comunicativas del inglés: escritura (writing), escucha (listening), lectura (reading) y habla (speaking). Desde una 
perspectiva comunicativa, son muchas las bondades -en términos de interacción- que promueve hacer el pensamiento visible.

Adicional a lo anterior, además de aportar nuevas herramientas que ayuden a promover el desarrollo de las habilidades comunicativas, como lo son las rutinas de pensamiento y los organizadores gráficos, también se abren nuevas oportunidades en la manera de pensar una clase de inglés, por ejemplo: 1) resaltando la importancia que tiene la lengua materna dentro del proceso de adquisición de una lengua extranjera; 2) enfatizando la necesidad de hacer visibles los nuevos conceptos e ideas para una mejor comprensión del contenido; y 3) subrayando la necesidad de estructurar la dinámica de la clase con el fin de desarrollar las habilidades comunicativas y los patrones de pensamiento que lleven a una comprensión gramatical y conceptual de manera más eficiente. En otras palabras, vincular el lenguaje con el desarrollo del pensamiento.

\section{Referencias}

Abbott, J. y Ryan, T. (1999). Constructing knowledge, reconstructing schooling. Educational Leadership, 57(3), 66-69.

Azcoaga, J. (1974). Aprendizaje fisiológico y aprendizaje pedagógico (1 ${ }^{a}$ ed.). Editorial Ateneo.

BBC News Mundo. (08 de septiembre de 2019). Cómo el inglés se convirtió en la lengua "universal" de la ciencia y por qué en el futuro puede dejar de serlo. BBC News Mundo. https://www.bbc.com/mundo/ noticias-49610224

Bloom, B. y Krathwohl, D. (1956). Taxonomy of educational objectives: the classification of educational goals (Handbook 1: cognitive domain). Longman, Green and Co Ltd.

Bonilla, C. y Rodríguez, P. (1997). Más allá de los métodos de la investigación en ciencias sociales ( $1^{\mathrm{a}}$ ed.). Editorial Norma.

Bruner, J., Wood, D. y Ross, G. (1987). The role of tutoring in problem solving. The Journal of Child Psychology and Psychiatry, 17(2), 89-100. https:// doi.org/10.1111/j.1469-7610.1976.tb00381.x

Castaño, J. (2003). Bases neurobiológicas del lenguaje y sus alteraciones. Revista de Neurología, 36(8), 781785. https://doi.org/10.33588/rn.3608.2002206
Dajani, M. (2016). Using Thinking Routines as a pedagogy for teaching english as a second language in Palestine. Journal of Education Research and Practice, 6(1), 1-18. https://doi.org/10.5590/JERAP.2016.06.1.01

Damasio, A. y Damasio, H. (1992). Brain and language. Scientific American, 267(3), 63-71. https://doi. org/10.1038/scientificamerican0992-88

Decreto 3870 de 2006. [MEN. Ministerio de Educación Nacional de Colombia]. Por el cual se reglamenta la organización y funcionamiento de los programas de educación para el trabajo y el desarrollo humano en el área de idiomas y se establecen las condiciones mínimas de calidad. 02 de noviembre de 2006. https://www. mineducacion.gov.co/1759/w3article-112277.html?_noredirect=1

Dehaene, S. (2019). ¿Cómo aprendemos? (1 $1^{\mathrm{a}}$ ed.). Siglo XXI Editores.

Díaz-Barriga, A. (2013). Guía para la elaboración de una secuencia didáctica. Universidad Nacional Autónoma de México (UNAM). http://www.setse. org.mx/ReformaEducativa/Rumbo\%20a\%20la\%20 Primera\%20Evaluaci\%C3\%B3n/Factores\%20de\%20 Evaluaci\%C3\%B3n/Pr\%C3\%A1ctica\%20Profesional/ Gu\%C3\%ADa-secuencias-didacticas_Angel\%20 D\%C3\%ADaz.pdf

EF Education First. (2019). EF EPI 2019. https://www. ef.com.co/epi/regions/latin-america/colombia/

El Tiempo. (17 de noviembre de 2019). Colombia, entre los países que se rajan en el dominio del inglés. El Tiempo. https://www.eltiempo.com/vida/educacion/ colombia-se-raja-en-nivel-de-ingles-segun-estudiode-ef-education-first-433880

Elliot, J. (1993). El cambio educativo desde la investigación-acción (4 ${ }^{\mathrm{a}}$ ed.). Ediciones Morata.

Fleta, M. (2006). Aprendizaje y técnicas de enseñanza del inglés en la escuela. Encuentro, 16, 51-62.

Flores, M. (2016) La globalización como fenómeno político, económico y social. ORBIS: Revista Científica de Ciencias Humanas, 34, 26-41.

Gardner, H. (2011). Frames of mind: the theory of multiple intelligences ( $2^{\mathrm{a}}$ ed.). Basic Books.

Gonzáles, F. (1996). Acerca de la metacognición. Revista Paradigma, 14-17(1-2), 109-135.

Gopnik, A., Meltzoff, A. y Kuhl, P. (1999). The scientist in the crib ( $1^{\mathrm{a}}$ ed.). William Morrow \& Co.

Hart, B. y Risley, T. (1995). Meaningful differences in the everyday experience of young American children. Paul H Brookes.

Hume, D. (2005). Tratado de la naturaleza humana $\left(4^{a}\right.$ ed.). Tecnos. 
Krashen, S. y Terrell, T. (1998). The natural approach. Language acquisition in the classroom. Prentice Hall Europe.

Kuhl, P. (1998). Mechanistic relationships between development and learning. John Wiley E Sons, Inc.

Kuhl, P. (2010). Brain mechanisms in early language acquisition. Neuron, 67(5), 713-727. https://doi. org/10.1016/j.neuron.2010.08.038

Ley 115 de 1994. Por la cual se expide la ley general de educación. 08 de febrero de 1994. D. O. No. 41214.

López, D. y Muñoz, M. (2003). Aprendizaje temprano de una segunda lengua. Encuentro: revista de investigación e innovación en la clase de idiomas, 13-14, 169-175.

Mayoral, P. (2016). Estratégias didácticas para la enseñanza del idioma inglés a niños de preescolar: el caso de un colegio en Colima, México [Tesis doctoral, Instituto Tecnológico y de Estudios Superiores de Occidente]. Repositorio Institucional del Instituto Tecnológico y de Estudios Superiores de Occidente. https://rei.iteso.mx/bitstream/ handle/11117/3790/TOG\%20Pedro\%20Mayoral. pdf? sequence $=3$

Méndez, G. (2002). Formación de profesores de inglés de escuelas preescolares y primarias [Tesis de maestría, Universidad de Colima]. Repositorio institucional de la Universidad de Colima, México. http://digeset.ucol. mx/tesis_posgrado/Pdf/Guadalupe $\% 20$ Socorro\%20 Mendez\%20Flores.pdf

Navarro, B. (2010). Adquisición de la primera y segunda lengua en aprendientes en edad infantil y adulta. Philologica Urcitana. Revista semestral de iniciación a la investigación en filología, 2(11), 115-128.

Pérez, M. y Villamizar, G. (2018). Propuesta para enseñar inglés en preescolar a partir del análisis realizado en dos instituciones educativas de Santander. $I+D$ Revista de Investigaciones, 12(2), 6-18. https://doi. org/10.33304/revinv.v12n2-2018001

Perkins, D. (1992). Smart Schools (1 ${ }^{\mathrm{a}}$ ed.). The Free Press.

Piaget, J. (2016). El nacimiento de la inteligencia del niño ( $1^{\mathrm{a}}$ ed.). Booket.

Piaget, J. (2017). Seis estudios de psicología (1ª ed.). Editorial Skla.
Piaget, J. (2018). La psicología de la inteligencia (1 ${ }^{a}$ ed.). Siglo XXI Editores.

Reyes, E. (2005). El aprendizaje de una lengua extranjera en el aula de educación infantil. Aula de infantil, 27, 5-8.

Richards, J. y Rodgers, T. (2014). Approaches and methods in language teaching ( $3^{\mathrm{a}}$ ed.). Cambridge University Press.

Ritchhart, R. (2002). Intellectual character ( $1^{\text {a }}$ ed.). Jossey-Bass.

Ritchhart, R. (2015). Creating cultures of thinking: the 8 forces we must master to truly transform our schools ( $1^{\mathrm{a}}$ ed.). Jossey-Bass.

Ritchhart, R., Church, M. y Morrison, K. (2011). Making thinking visible ( $1^{\mathrm{a}}$ ed.). Jossey-Bass.

Salmon, A. (2008). Young English language learners making thinking and language visible. Colombian Applied Linguistics Journal, 10, 126-141. https:// doi.org/10.14483/22487085.101

Serrano, J. y Pons, R. (2011). El constructivismo hoy: enfoques constructivistas en educación. Revista Electrónica de Investigación Educativa, 13(1), 1-27.

Statista. (2020). The most spoken languages worldwide in 2019. https://www.statista.com/statistics/266808/ the-most-spoken-languages-worldwide/

Súarez, C. (2014) Entorn de l'origen de la teoria sociocultural: la zona de desenrotllament pròxim en la vida de Vigotsky. Anuari de Psicologia de la Societat Valenciana de Psicologia, 15(2), 179-192.

Taylor, S. y Bogdan, R. (1987) Introducción a los métodos cualitativos de investigación. Ediciones Paidós Ibérica, S.A.

Verdejo-García, A. y Bechara, A. (2010). Neuropsicología de las funciones ejecutivas. Psicothema, 22(2), 227235.

Vygotsky, L. (1978). Mind in society: the development of higher psychological processes ( $7^{\mathrm{a}}$ ed.). Harvard University Press.

Vygotsky, L. (2015). Pensamiento y lenguaje (1 ${ }^{\mathrm{a}}$ ed.). Booket.

Vygotsky, L. (2018). El desarrollo de los procesos psicológicos superiores ( $4^{\mathrm{a}} \mathrm{ed}$.). Austral. 